\title{
DESAFIOS COGNITIVOS DA REPRESENTAÇÃO DA OFERTA E DA DEMANDA EM GRÁFICOS ELABORADOS POR ESTUDANTES UNIVERSITÁRIOS
}

Ruth M. Hofmann ${ }^{1}$

\section{RESUMO}

Este trabalho analisa registros de representação gráfica de oferta e demanda construídos por estudantes de Engenharia, no intuito de identificar desafios cognitivos subjacentes ao ensino de Economia. Trata-se de uma pesquisa exploratória que utilizou gráficos elaborados por 47 alunos durante um exercício realizado em uma aula de Economia. Dentre os resultados obtidos, podem-se destacar a falta de familiaridade com a estática comparativa enquanto método de análise econômica e a dificuldade conceitual e gráfica inerente às distinções entre demanda e quantidade demandada, oferta e quantidade ofertada. Os resultados encontram respaldo empírico em trabalhos relacionados à literatura acerca dos desafios cognitivos subjacentes à interpretação de gráficos. Os desafios cognitivos observados referem-se a falhas de codificação, de rotulagem e de processamento de informação, por exemplo.

Palavras-chave: Ensino de Economia. Estática comparativa. Erro de rotulagem.

\section{Como citar este documento - ABNT}

HOFMANN, Ruth M. Desafios cognitivos da representação da oferta e da demanda em gráficos elaborados por estudantes universitários. Revista Docência do Ensino Superior, Belo Horizonte, v. 10, e015867, p. 1-20, 2020 DOI: https://doi.org/10.35699/2237-5864.2020.15867.

Recebido em: 07/11/2019 Aprovado em: 20/01/2020 Publicado em: 17/04/2020

\footnotetext{
${ }^{1}$ Universidade Federal do Paraná (UFPR), Curitiba, PR, Brasil. ORCID ID: https://orcid.org/0000-0001-9495-5411. E-mail: ruthofmann@gmail.com.
} 


\section{DESAFÍOS COGNITIVOS DE LA REPRESENTACIÓN DE LA OFERTA Y LA DEMANDA EN GRÁFICOS PREPARADOS POR ESTUDIANTES UNIVERSITARIOS}

\section{RESUMEN}

Este artículo analiza los gráficos de oferta y demanda construidos por alumnos de Ingeniería para identificar los desafíos cognitivos que subyacen a la enseñanza de la economía. Esta es una investigación exploratoria que utilizó los gráficos preparados por 47 alumnos durante un ejercicio en una clase de Economía. Entre los resultados podemos destacar la falta de familiaridad con la estática comparativa como método de análisis económico y la dificultad conceptual y gráfica inherente a las distinciones entre demanda y cantidad demandada, oferta y cantidad ofrecida. Los resultados encuentran apoyo empírico en trabajos relacionados con la literatura sobre los desafíos cognitivos que subyacen a la interpretación de los gráficos. Los desafíos cognitivos observados se relacionan con fallas de codificación, errores de calificación y procesamiento de información, por ejemplo.

Palabras clave: Enseñanza de la economía. Estática comparativa. Error de calificación.

\section{COGNITIVE CHALLENGES OF THE REPRESENTATION OF SUPPLY AND DEMAND IN GRAPHICS PREPARED BY UNIVERSITY STUDENTS}

\section{ABSTRACT}

This work analyzes records of graphical representation of supply and demand built by Engineering students, in order to identify cognitive challenges underlying the teaching of Economics. It is about an exploratory research that used graphics prepared by 47 students during an exercise carried out in an Economics class. Among the obtained results, it is possible to highlight the lack of familiarity with comparative statics as a method of economic analysis and the conceptual and graphic difficulty inherent to the distinctions between: demand and quantity demanded, supply and quantity offered. The results find an empirical support in related works in the literature about the cognitive challenges underlying the interpretation of graphs. The cognitive challenges observed refer to failures in coding, labeling and information processing, for example.

Keywords: Economics Teaching. Comparative Statics. Labeling Error. 


\section{INTRODUÇÃO}

Gráficos, figuras e tabelas são recursos didáticos recorrentemente utilizados na divulgação de conhecimento científico, podendo tais recursos facilitar ou prejudicar o processo de interpretação de informações relevantes (FRANZBLAU; CHUNG, 2012). Ilustrar graficamente relações entre variáveis no intuito de explicar teorias, hipóteses e modelos é prática compartilhada por diferentes áreas do conhecimento, havendo ampla variedade de tipos, formatos e representações gráficas à disposição de pesquisadores e professores no processo de divulgação da ciência. Podem-se encontrar, na literatura, diferentes abordagens para analisar os desafios de compreensão e construção de gráficos (CARSWELL; EMERY; LONON, 1993; ZACKS; TVERSKY, 1999; SHAH; MAYER; HEGARTY, 1999).

Na condição de Ciência Social Aplicada, a Economia recorre a teorias e modelos para analisar, explicar e prever os mais variados fenômenos econômicos. A construção dos modelos, com suas respectivas representações algébricas, gráficas e mesmo textuais, não é fato trivial. Nem sempre elementos do senso comum permitem que eventos econômicos sejam facilmente compreendidos. Nessa perspectiva, pode-se pressupor que o ensino da Economia como disciplina científica seja um campo potencialmente profícuo para a análise de processos cognitivos associados à construção de representações gráficas. O emprego sistemático de gráficos de oferta e demanda é, por si só, território abrangente para exploração didática e epistemológica de tais registros. Especificamente no âmbito do ensino, analisar erros cometidos por estudantes na elaboração de gráficos de oferta e demanda pode proporcionar elementos para o aprimoramento do ensino e para a compreensão de processos cognitivos envolvidos na construção de gráficos.

Nesse contexto, o objetivo deste artigo consiste em analisar os registros gráficos de oferta e demanda construídos por estudantes de Engenharia, identificando os principais tipos de erros cometidos. Os resultados são analisados à luz da literatura acerca dos desafios cognitivos da compreensão de gráficos.

\section{OS DESAFIOS COGNITIVOS DA COMPREENSÃO DE GRÁFICOS}

A preocupação com os processos cognitivos subjacentes à interpretação e à compreensão de gráficos tem se situado, sobretudo, no contexto do letramento gráfico, expressão que designa um conjunto de competências e habilidades necessárias à leitura de gráficos (SOFO, 1985; ROBERTS; BRUGAR, 2017; GALESIC; GARCIA-RETAMERO, 2011; READENCE; BEAN; BALDWIN, 2004).

Leinhardt et al. (1990) realizam uma minuciosa revisão de literatura sobre ensino e aprendizagem de gráficos a partir de temas como a tarefa e sua apresentação, o aprendiz e o desenvolvimento da compreensão sobre gráficos e funções. Especificamente no que se refere 
Ruth M. Hofmann

à tarefa, Leinhardt et al. (1990) organizam a revisão de literatura a partir de quatro elementos. O primeiro elemento é a ação do estudante ou aprendiz e refere-se à interpretação ("leitura", ou "fazer sentido") ou à construção do gráfico. A construção, por sua vez, pode ser uma atividade na qual se propõe que um gráfico seja plotado a partir de um conjunto de dados, pode ser a determinação de uma equação a partir de um gráfico ou mesmo produzir um exemplo de uma função. Construir, nesse contexto, significa gerar algo novo a partir de um conjunto de dados ou de uma função abstrata. O segundo elemento é a situação (ou contexto) do gráfico e refere-se ao fato de o gráfico estar mais contextualizado ou menos contextualizado (abstrato). A natureza da situação pode influenciar na plausibilidade dos resultados e no tipo de variável utilizado na tarefa. O terceiro elemento inclui as variáveis e sua natureza: trata-se dos objetos das funções e gráficos, sejam concretos ou abstratos. As propriedades das unidades são relevantes nessa perspectiva (sejam categorias, intervalos, taxas, etc., ou ainda, os dados podem ser discretos ou contínuos). O quarto elemento, por fim, é a localização da atenção em uma tarefa específica, liga a situação e a ação. Pode voltar-se a coordenar o gráfico e seus componentes ou pode voltar-se a coordenar os eixos, seus rótulos e suas escalas.

No que concerne ao aprendizado, Leinhardt et al. (1990) discutem, sobretudo, intuições e conceitos errados dos estudantes. As intuições são definidas como as características do conhecimento que emergem da experiência cotidiana, ainda que no caso de alguns estudantes possa envolver uma composição entre conhecimento cotidiano e uma compreensão mais profunda do conhecimento formal (LEINHARDT et al., 1990, p. 24). Os conceitos errados são definidos como características incorretas do conhecimento dos estudantes que são repetíveis e explícitas (LEINHARDT et al., 1990, p. 30). Intuições e conceitos errados podem levar à interpretação e à construção errada de representações gráficas.

À luz de um referencial calcado no processamento de informações, Postigo e Pozo (2000) discutem a leitura e a aprendizagem de gráficos a partir de três níveis. O primeiro seria o da informação explícita. Trata-se do nível mais superficial de leitura de gráficos, envolvendo a identificação de seus elementos constituintes (título, número, nome e tipo das variáveis, por exemplo). O segundo seria o da informação implícita. Trata-se de um nível de processamento que requer uma leitura capaz de identificar relações intra e intervariáveis. Além disso, a aprendizagem da informação implícita requer algum conhecimento acerca de convenções gráficas e processos de decodificação de símbolos. A eventual necessidade de tradução da informação de um código em outro também caracteriza o nível de processamento da informação implícita. O terceiro seria o nível da informação conceitual, o nível de aprendizagem essencialmente caracterizado pelo estabelecimento de relações conceituais a partir da análise global da estrutura gráfica. Faz-se necessário recorrer a outros 
Ruth M. Hofmann

conhecimentos disponíveis, relacionados ao conteúdo do gráfico, para que interpretações, explicações e previsões sobre os fenômenos representados no gráfico sejam realizadas.

A classificação dos níveis proposta por Postigo e Pozo (2000) é utilizada, por Camargo Filho, Laburu e Barros (2011), para analisar dificuldades semióticas subjacentes à construção de gráficos cartesianos em cinemática, e, por García García e Perales Palacíos (2005), para analisar o desempenho de estudantes de graduação de química em tarefas de interpretação de gráficos cartesianos.

Para Shah e Hoeffner (2002), os desafios cognitivos subjacentes à compreensão e interpretação de gráficos podem estar relacionados a três fatores principais: características visuais do gráfico (a exemplo do tipo, formato, tamanho, cor e legenda do gráfico); o conhecimento que o leitor tem sobre gráficos; e o conhecimento e as expectativas sobre o conteúdo dos dados do gráfico. No que se refere às características visuais, menciona-se o fato de que, quando se deparam com gráficos bidimensionais de linha, os leitores estão mais inclinados a descrever tendências do tipo $x-y$ (por exemplo, enquanto $x$ aumenta, y reduz) do que quando se deparam com gráficos de barras. (CARSWELL; EMERY; LONON, 1993; ZACKS; TVERSKY, 1999; SHAH et al., 1999). No que se refere ao conhecimento dos gráficos, os leitores tipicamente esperam variáveis dependentes plotadas como função de y em um gráfico de linha, e que as variáveis causais ou independentes sejam plotadas como função do eixo y. Quando a expectativa é frustrada (um gráfico que viola o formato esperado), erros de interpretação podem ocorrer (GATTIS; HOLYOAK, 1996).

No que se refere ao conhecimento do conteúdo do gráfico, quando o conhecimento prévio do leitor entra em conflito com a informação ali contida, podem ocorrer erros sistemáticos de interpretação (SHAH, 1995).

O tipo de dado pode afetar estimativas quantitativas e interpretações qualitativas dos leitores. A mudança parece ser particularmente difícil de ser interpretada graficamente. Variáveis como crescimento, velocidade e aceleração contêm certa complexidade inerente à interpretação gráfica (a velocidade, por exemplo, é mais facilmente compreendida do que a aceleração). Estudantes lidam mais facilmente com gráficos nos quais o tempo é uma dimensão expressa. Uma das hipóteses para a maior facilidade, nesses casos, é a utilização recorrente do tempo em situações didáticas nas quais o contexto apresentado depende do tempo (SHAH; HOEFFNER, 2002).

Especificamente no que se refere aos gráficos de linha, três tipos de processo são particularmente relevantes para a interpretação (SHAH; CARPENTER, 1995). O primeiro consiste em codificar os padrões visuais (se há múltiplas linhas, se as linhas são paralelas, convergentes, se elas se interceptam, etc.). O segundo consiste em traduzir características visuais em relações conceituais nelas representadas. Requer associação entre conhecimento 
Ruth M. Hofmann

quantitativo e padrões visuais. Quando os padrões visuais evocam conceitos quantitativos adequados, a compreensão do gráfico é fácil. O terceiro processo consiste em determinar os referentes dos conceitos que estão sendo quantificados e associá-los àqueles das funções codificadas. Para tanto, é necessário ler as legendas e os valores dos eixos e relacioná-los às linhas correspondentes e aos pontos de dados.

Shah e Carpenter (1995) identificaram um conjunto de erros comuns cometidos por estudantes em tarefas específicas de construção de gráficos de linhas. O primeiro tipo seriam os erros de simplificação, ou tendência para desenhar gráficos mais simétricos, mais achatados ou mais paralelos do que o correto. Tal tendência consiste em uma falha potencialmente decorrente de limitações na memória de informação gráfica ou de representações internas que incluem um conjunto presumido de propriedades sobre relações entre variáveis do tipo $x-y$. $O$ segundo tipo seriam falhas de codificação ou informação incompleta sobre propriedades métricas das variáveis. Pode ser mais comum se a característica visual da informação não for visualmente saliente, se os gráficos forem muito complexos ou se a apresentação dos gráficos aos estudantes levar a ilusões visuais. Não é um tipo de erro comum para gráficos simples. A maior fonte de erro e incompletude decorre da falha em recuperar o conhecimento conceitual sobre a interpretação quantitativa de um padrão codificado. O terceiro tipo seriam falhas de rotulagem, ou erros de rotulagem (indicação de legenda), que podem ocorrer porque os sujeitos da pesquisa não codificam ou não lembram como os valores na legenda correspondem às linhas individuais. Tais erros são comuns no desenho de gráficos com três eixos $(x-y-z)$. O quarto tipo seria o erro geral, caracterizado pela dificuldade de traduzir informações gráficas ou pela violação de convenções gráficas elementares. Trata-se de erros gerais decorrentes de inferências equivocadas ou mesmo erros que violam grosseiramente convenções gráficas (desenhar linhas com comprimentos desproporcionais ou atribuir múltiplos rótulos a uma mesma linha, por exemplo).

Shah e Hoeffner (2002) concluíram que algumas perguntas precisariam de respostas no âmbito das investigações sobre a compreensão de gráficos. Considerando que, até então, as pesquisas cognitivas sobre gráficos focavam na compreensão ao invés da construção, os questionamentos incluiriam, por exemplo: "quais os tipos de erros cometidos na construção de gráficos?" e "os estudantes escolhem diferentes tipos de gráficos dependendo do objetivo (comunicar fatos específicos, testar hipóteses etc.) ou eles estão enviesados a usar formatos familiares?". Tais questionamentos foram ponderados neste artigo. 
Ruth M. Hofmann

\section{OFERTA E DEMANDA COMO OBJETOS DE ENSINO: DEFINIÇÕES E REPRESENTAÇÕES EM MANUAIS DE INTRODUÇÃO À ECONOMIA}

Gráficos bidimensionais compõem o instrumental básico de análise econômica. Sendo utilizado para visualizar o comportamento de variáveis, como taxas de crescimento do Produto Interno Bruto (PIB), inflação, exportações ou importações, o plano cartesiano é o mais comumente utilizado na Economia. Trata-se de uma ferramenta importante para acompanhar o comportamento e a evolução de variáveis de interesse teórico ou pragmático. A preferência pelos gráficos bidimensionais do plano cartesiano justifica-se, essencialmente, pela tradição e pela facilidade proporcionada na visualização da relação entre variáveis (ESTRADA, 2010).

Especificamente no que se refere ao ensino de Economia, a análise gráfica é particularmente relevante para que sejam exploradas noções como oferta, demanda e equilíbrio de mercado, no âmbito da Microeconomia. O roteiro didático tradicionalmente empregado por manuais de economia, para que tais conceitos sejam lecionados, inicia-se com definições necessárias para a compreensão das respectivas representações gráficas (PARKIN, 2009; PASSOS; NOGAMI, 2016; VASCONCELLOS, 2010; MOCHÓN, 2007).

O ensino de conceitos de Microeconomia pressupõe que os estudantes sejam apresentados à definição de demanda individual e de mercado, sendo a última o somatório das demandas individuais para cada nível de preço. A definição de demanda faz-se acompanhar de seus respectivos determinantes, sendo necessária a distinção entre demanda (a curva em si) e quantidade demandada (um ponto específico da curva de demanda que relaciona preços e quantidades). Tal distinção é essencial para a análise das mudanças na demanda e consequentes mudanças no equilíbrio de mercado (PASSOS; NOGAMI, 2016).

Enquanto a demanda representa em essência o consumidor, a oferta representa essencialmente os produtores (firmas, na terminologia acadêmica). Assim como na apresentação da definição de demanda, faz-se necessário definir a oferta (individual e de mercado), apresentar seus respectivos determinantes e a diferença gráfica e conceitual entre oferta (a curva em si) e a quantidade ofertada (necessária à análise das mudanças no equilíbrio de mercado). Definições de demanda e de oferta antecedem a apresentação da "lei geral da demanda", com suas exceções, e da "lei geral da oferta", ambas essenciais para a representação gráfica do equilíbrio de mercado. A lei geral da demanda garante a inclinação negativa da curva de demanda quando é plotada num plano cartesiano no qual quantidades demandadas são representadas no eixo das abscissas ("eixo x") e os preços são representados no eixo das ordenadas ("eixo $y$ "). A lei geral da oferta, por sua vez, garante a inclinação positiva da curva de oferta quando é plotada no mesmo plano cartesiano caracterizado para a demanda (quantidades representadas nas abscissas e preços nas ordenadas) (PARKIN, 2009). 
Ruth M. Hofmann

A análise das mudanças no equilíbrio de mercado é desenvolvida a partir de uma premissa simplificadora, a cláusula coeteris paribus, expressão em latim para "tudo o mais constante". Trata-se de um recurso didático que tem como objetivo analisar o efeito individualizado de mudanças que variáveis específicas podem ter sobre o equilíbrio de mercado (o equilíbrio, como definição, ocorre quando a demanda se iguala à oferta, ou seja, quando as curvas se interceptam, não havendo assim excesso de demanda nem de oferta). O objetivo é "isolar" analiticamente o efeito que determinantes da demanda e da oferta podem ter sobre o preço e quantidade de equilíbrio (MOCHÓN, 2007).

Conceitual e graficamente, mudanças das quantidades demandadas e ofertadas são diferentes de mudanças da demanda e da oferta, tal como ilustrado na Figura 1. Mudanças são expressas no que diz respeito a estática comparativa, recurso metodológico que não inclui o tempo como variável explícita do gráfico. Não se trata, portanto, de gráficos de fluxo (ou evolução) de uma variável - seja preço, seja quantidade - em relação a unidades específicas de tempo (semana, mês ou ano, por exemplo). O "antes" e o "depois" são graficamente representados mediante denominação distinta das curvas.

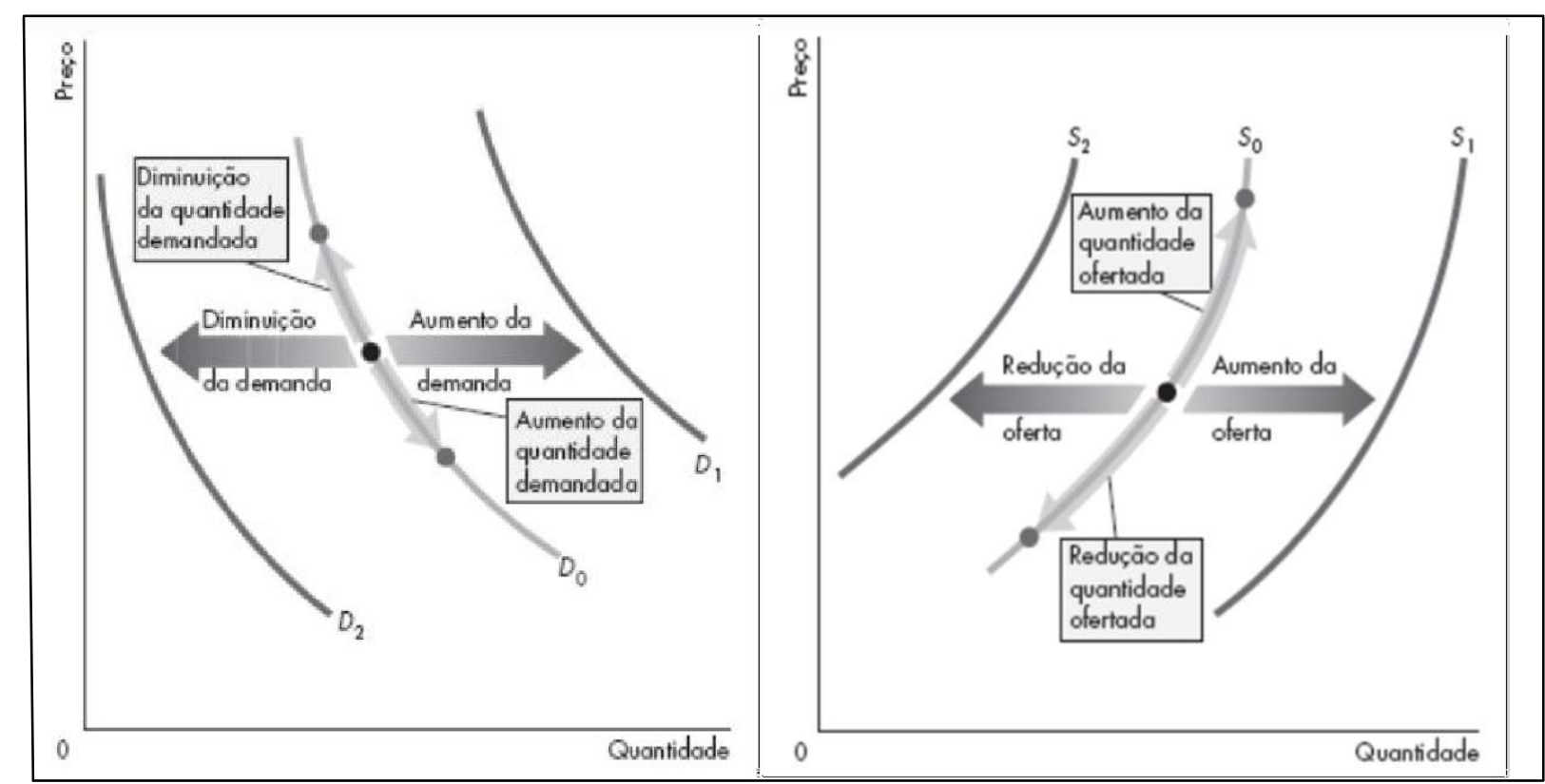

Figura 1 - Diferenças entre mudanças das quantidades (demandada e ofertada) e mudanças da demanda e da oferta

Fonte: (PARKIN, 2009, p. 59 e 63).

A clareza da distinção entre quantidade demandada e demanda, entre quantidade ofertada e oferta é importante para identificar excesso de demanda e excesso de oferta (Figura 2), situações nas quais o mecanismo de preços da economia se torna relevante para proporcionar o equilíbrio de mercado. 


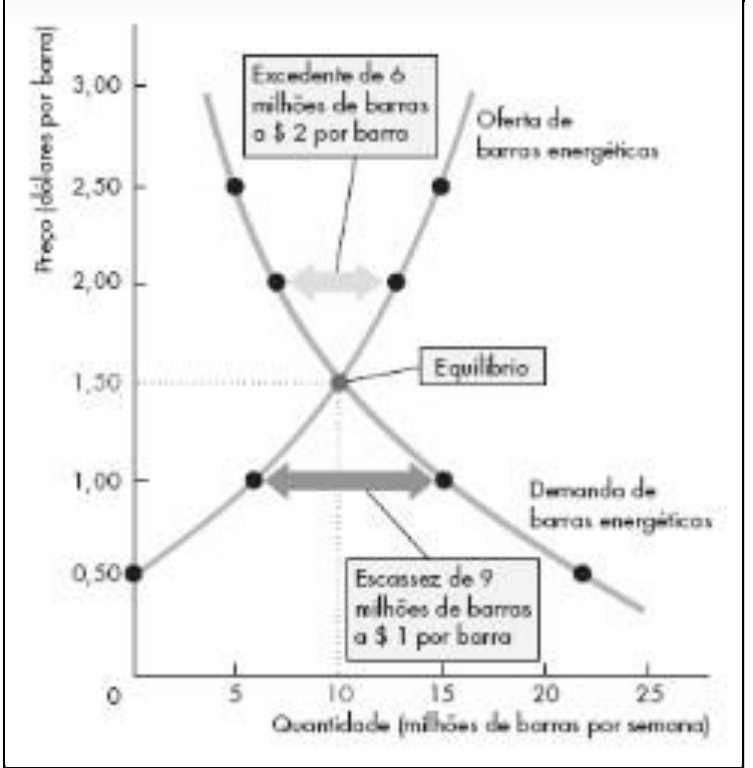

Figura 2 - Representação gráfica do equilíbrio de mercado Fonte: (PARKIN, 2009, p. 64).

As mudanças no equilíbrio, assim, são decorrentes de mudanças na demanda ou na oferta, ambas sendo representadas por deslocamentos das curvas, tal como ilustrado na Figura 3.
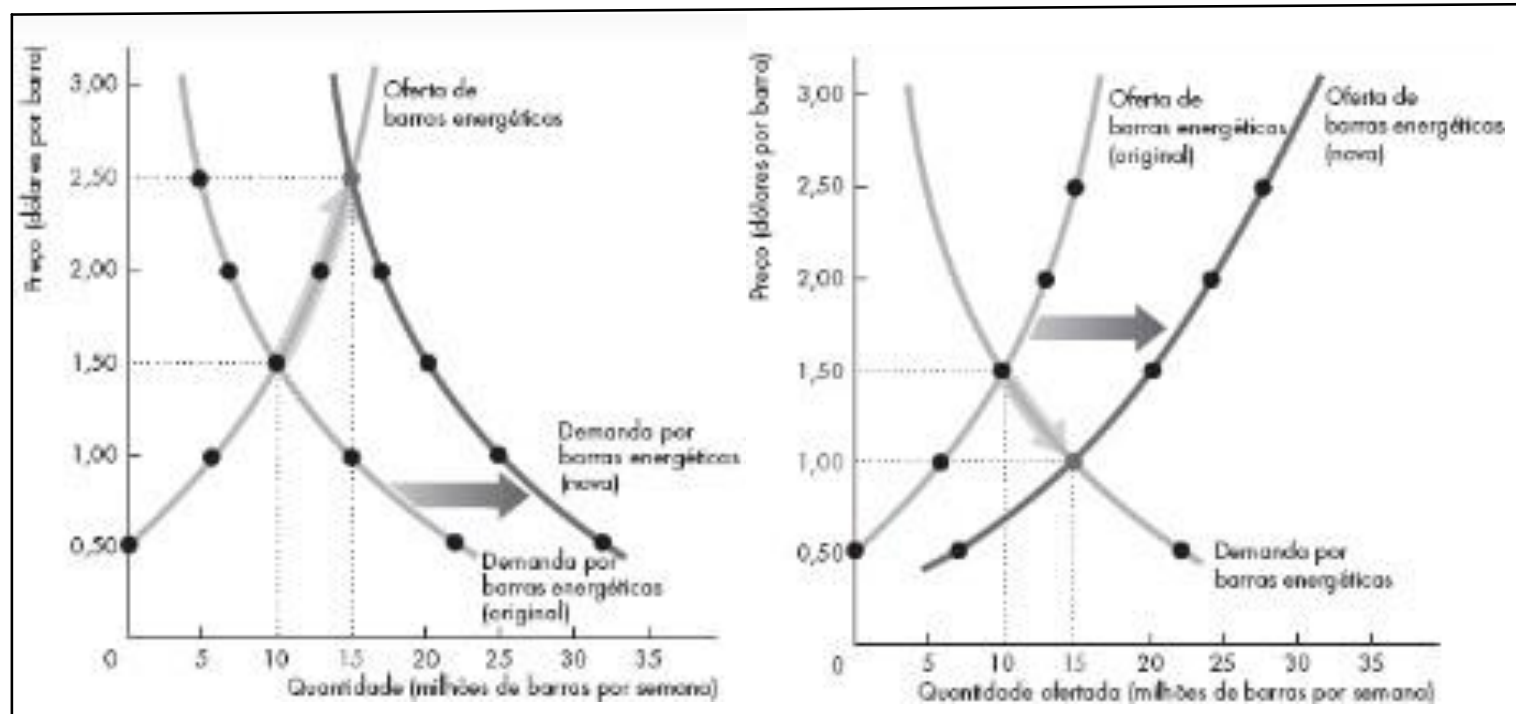

Figura 3 - Representação gráfica de mudanças no equilíbrio de mercado provocadas por mudanças na demanda e na oferta

Fonte: (PARKIN, 2009, p. 65 e 66).

A análise gráfica de mudanças no equilíbrio de mercado comumente antecede a determinação matemática do equilíbrio em manuais de introdução à Economia. Em alguns casos (PASSOS; NOGAMI, 2016), a análise algébrica consta como apêndice do capítulo "Demanda, Oferta e Equilíbrio de Mercado". 
Ruth M. Hofmann

Para identificar o preço e a quantidade de equilíbrio de determinando bem ou serviço algebricamente, deve-se "igualar" ambas funções (demanda e oferta) e "isolar" a variável preço, supondo-se "mantidas constantes as demais variáveis" (coeteris paribus). O resultado do cálculo é o "preço de equilíbrio". Tendo-se o valor correspondente ao preço, para identificar a quantidade de equilíbrio, insere-se o preço na equação da demanda (ou da oferta, pois a quantidade de equilíbrio supõe igualdade entre ambas), obtendo-se a quantidade de equilíbrio.

Ainda que em manuais de introdução à Economia nem sempre a discussão seja aprofundada, a determinação simultânea de preço e quantidade no modelo de interação entre oferta e demanda é pormenorizado, em disciplinas como Econometria, no contexto das "equações simultâneas" e da endogeneidade de variáveis. Não se trata de assuntos normalmente abordados quando a Economia é disciplina introdutória em determinados cursos de graduação.

\section{MATERIAIS E MÉTODOS}

Os registros analisados no presente artigo são resultado de um exercício realizado durante uma aula de Economia, ministrada para um curso de graduação em Engenharia, ofertado por uma universidade pública do sul do Brasil. Trata-se de uma pesquisa essencialmente exploratória. À luz da literatura abordada, em especial no que reporta à classificação de Leinhardt et al. (1990), pode-se afirmar que a ação do estudante consistiu na construção de um gráfico a partir de funções abstratas de oferta e demanda. O contexto da tarefa consistiu em uma situação didática, e a situação do gráfico foi dada pelo enunciado que, não de todo abstrato, também não teve por base dados numéricos específicos. No que diz respeito a variáveis e sua natureza, os objetos das funções e dos gráficos pressupunham certa abstração e, como suposição básica de representações de oferta e demanda, os dados deveriam ser assumidos como contínuos (simplificação inerente ao exercício teórico em economia). O foco deveria ser direcionado à coordenação do gráfico, de seus componentes, eixos e rótulos. 0 enunciado da atividade consta no Quadro 1.

\footnotetext{
Represente graficamente e explique:

a. Os efeitos do aumento do preço do cacau sobre o equilíbrio de mercado de chocolate.

b. Os efeitos sobre o equilíbrio de mercado de transporte público (ônibus) decorrentes da entrada de empresas como o Uber no setor.

c. Os efeitos sobre o equilíbrio de mercado de cerveja decorrentes da divulgação de um estudo indicando que álcool aumenta o risco de câncer.

d. Os efeitos do aumento da renda do consumidor sobre o equilíbrio de mercado de: (i) Pacotes de viagem; (ii) Sal; (iii) Transporte público (passagens de ônibus).

Quadro 1 - Enunciado da atividade proposta

Fonte: elaborado pela autora.
} 
Ruth M. Hofmann

A atividade foi proposta depois de duas aulas sobre representações gráficas de equilíbrio de mercado e de mudanças no equilíbrio de mercado. Cada aula foi realizada em dias diferentes, com duração média de uma hora e trinta minutos. A exposição do conteúdo contemplou a ilustração (no quadro negro, com giz colorido) de situações diversas que promoveriam mudanças na oferta e na demanda (deslocamentos positivos e negativos em ambas). Participaram da atividade 47 estudantes dentre 55 matriculados e frequentes na disciplina durante o primeiro semestre de 2019.

É importante notar que a expectativa pedagógica acerca das respostas elaboradas pelos estudantes se pautou pela reprodução dos gráficos apresentados nas duas aulas anteriores. Depois de ministrado o conteúdo, esperava-se que os estudantes fossem capazes de reproduzir os gráficos apresentados, sobretudo os deslocamentos das curvas de oferta e demanda (com seus respectivos efeitos sobre o preço e a quantidade de equilíbrio).

\section{RESULTADOS E DISCUSSÃO}

Dentre 47 estudantes que participaram da atividade, oito reproduziram corretamente todos os gráficos, conforme Figura 4, que registra a redução da demanda por cerveja alterando o equilíbrio de mercado depois da divulgação de um estudo indicando que álcool aumenta o risco de câncer:

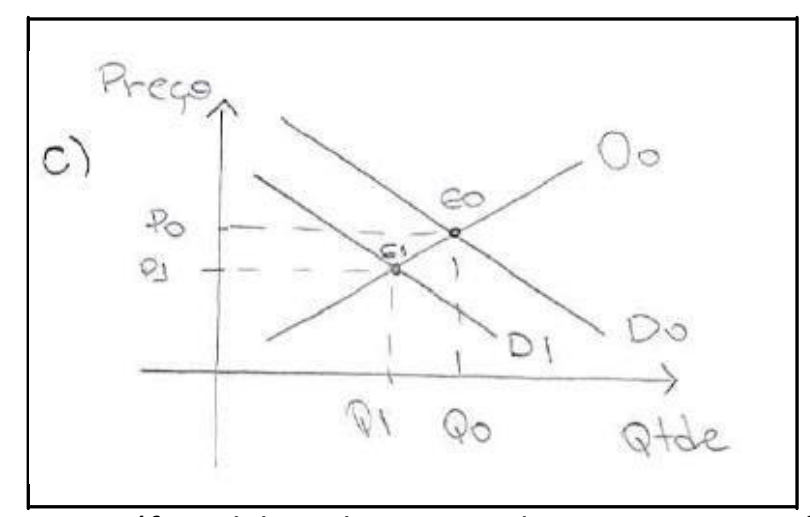

Figura 4 - Exemplo de registro gráfico elaborado por estudante: registro conforme expectativa Fonte: elaborada por estudante.

Os equívocos cometidos pelos alunos na execução da tarefa foram agrupados, por similaridade, em 9 tipos, sendo sua frequência mensurada em termos da quantidade de alunos que os cometeram. Cabe notar que um mesmo estudante pode ter cometido mais de um tipo de erro, motivo pelo qual a frequência somada não corresponde ao tamanho da amostra. Os dados são apresentados na Tabela 1. 


\begin{tabular}{llcc}
\hline \multicolumn{1}{c}{ Tipo de erro } & $\begin{array}{c}\text { Número } \\
\text { de alunos }\end{array}$ & $\begin{array}{c}\text { Percentual em } \\
\text { relação ao total de } \\
\text { alunos }\end{array}$ \\
\hline 1 Ausência de aderência com o & 17 & $36 \%$ \\
2 & Deslocamento da curva errada & 9 & $19 \%$ \\
3 & Ausência de rótulos para os eixos & 4 & $9 \%$ \\
4 & Inversão dos eixos & 4 & $9 \%$ \\
5 & Ausência de qualquer registro & 2 & $4 \%$ \\
6 & Inversão da inclinação das curvas & 2 & $4 \%$ \\
7 & Ausência de denominação das & 1 & $2 \%$ \\
8 & Ausência de uma das curvas & 1 & $2 \%$ \\
9 & Ausência de deslocamento & 1 & $2 \%$ \\
\hline
\end{tabular}

Tabela 1 - Tipos de erros identificados

Fonte: elaborada pela autora.

O Quadro 2 relaciona os erros identificados com a revisão de literatura e com os conceitos microeconômicos que deveriam ser mobilizados durante a atividade.

\begin{tabular}{|l|l|l|}
\hline $\begin{array}{l}\text { Tipo de erro } \\
\text { identificado }\end{array}$ & \multicolumn{1}{|c|}{ Correspondência na literatura } & \multicolumn{1}{c|}{ Conceito econômico } \\
\hline $\begin{array}{l}\text { Ausência de } \\
\text { aderência } \\
\text { com o modelo }\end{array}$ & $\begin{array}{l}\text { - Erros de simplificação, falhas de } \\
\text { codificação, falhas de rotulagem e erros } \\
\text { gerais (SHAH; CARPENTER, 1995). } \\
\text { - Intuições e conceitos equivocados } \\
\text { (LEINHARDT et al. 1990). } \\
\text { - Dificuldades de processamento da } \\
\text { informação explícita e principalmente } \\
\text { implícita (POSTIGO; POZO, 2000). }\end{array}$ & $\begin{array}{l}\text { - Falta de domínio global (conceitual e } \\
\text { gráfico) das noções de demanda e oferta } \\
\text { associada à falta de compreensão de suas } \\
\text { leis e determinantes. } \\
\text { - Falta de compreensão do equilíbrio de } \\
\text { mercado, de mudanças no equilíbrio e do } \\
\text { próprio mecanismo de preços. }\end{array}$ \\
\hline $\begin{array}{l}\text { Deslocamento } \\
\text { da curva } \\
\text { errada e } \\
\text { deslocamento } \\
\text { de duas } \\
\text { curvas }\end{array}$ & $\begin{array}{l}\text { - Falhas de codificação e falhas de } \\
\text { rotulagem (SHAH; CARPENTER, 1995). } \\
\text { - Dificuldades de processamento da } \\
\text { informação implícita e conceitual } \\
\text { (POSTIGO; POZO, 2000). }\end{array}$ & $\begin{array}{l}\text { - Falta de domínio dos conceitos de } \\
\text { oferta e demanda e, sobretudo, de seus } \\
\text { respectivos determinantes. } \\
\text { - Incapacidade de distinção da } \\
\text { independência analítica entre oferta e } \\
\text { demanda. } \\
\text { - Falta de compreensão da clausula } \\
\text { coeteris paribus. }\end{array}$ \\
\hline $\begin{array}{l}\text { Ausência de } \\
\text { rótulos para } \\
\text { os eixos }\end{array}$ & $\begin{array}{l}\text { - Falhas de rotulagem e erro geral } \\
\text { (SHAH, CARPENTER, 1995). } \\
\text { - Dificuldades de processamento da } \\
\text { informação implícita, explícita e } \\
\text { conceitual (POSTIGO; POZO, 2000). }\end{array}$ & $\begin{array}{l}\text { - Falta de domínio da relação entre preço } \\
\text { e quantidade definida pelas leis de oferta } \\
\text { e demanda. }\end{array}$ \\
\hline $\begin{array}{l}\text { Inversão dos } \\
\text { eixos }\end{array}$ & $\begin{array}{l}\text { - Falhas de codificação e falhas de } \\
\text { rotulagem (SHAH; CARPENTER, 1995). } \\
\text { - Dificuldades de processamento da } \\
\text { informação implícita, explícita e } \\
\text { conceitual (POSTIGO; POZO, 2000). }\end{array}$ & $\begin{array}{l}\text { - Falta de domínio da relação entre preço } \\
\text { e quantidade definida pelas leis de oferta } \\
\text { e demanda. }\end{array}$ \\
\hline
\end{tabular}


Desafios cognitivos da representação da oferta e da demanda em gráficos elaborados por estudantes universitários

Ruth M. Hofmann

\begin{tabular}{|c|c|c|}
\hline $\begin{array}{l}\text { Tipo de erro } \\
\text { identificado }\end{array}$ & Correspondência na literatura & Conceito econômico \\
\hline $\begin{array}{l}\text { Ausência de } \\
\text { qualquer } \\
\text { registro } \\
\text { gráfico }\end{array}$ & $\begin{array}{l}\text { - Incapacidade de codificação (SHAH; } \\
\text { CARPENTER, 1995). } \\
\text { - Dificuldades de processamento da } \\
\text { informação conceitual, sobretudo } \\
\text { (POSTIGO; POZO, 2000). }\end{array}$ & $\begin{array}{l}\text { - Falta de domínio global (conceitual e } \\
\text { gráfico) das noções de demanda e oferta } \\
\text { associada à falta de compreensão de suas } \\
\text { leis e determinantes. } \\
\text { - Falta de compreensão do equilíbrio de } \\
\text { mercado, de mudanças no equilíbrio e do } \\
\text { próprio mecanismo de preços. }\end{array}$ \\
\hline $\begin{array}{l}\text { Inversão da } \\
\text { inclinação das } \\
\text { curvas }\end{array}$ & $\begin{array}{l}\text { - Falhas de codificação e falhas de } \\
\text { rotulagem (SHAH; CARPENTER, 1995). } \\
\text { - Dificuldades de processamento da } \\
\text { informação implícita, explícita e } \\
\text { conceitual (POSTIGO; POZO, 2000). }\end{array}$ & $\begin{array}{l}\text { - Falta de domínio da relação entre preço } \\
\text { e quantidade definida pelas leis de oferta } \\
\text { e demanda. }\end{array}$ \\
\hline $\begin{array}{l}\text { Ausência de } \\
\text { denominação } \\
\text { das curvas }\end{array}$ & $\begin{array}{l}\text { - Falhas de codificação e falhas de } \\
\text { rotulagem (SHAH; CARPENTER, 1995). } \\
\text { - Dificuldades de processamento da } \\
\text { informação implícita, explícita e } \\
\text { conceitual (POSTIGO; POZO, 2000). }\end{array}$ & $\begin{array}{l}\text { - Falta de domínio da relação entre preço } \\
\text { e quantidade definida pelas leis de oferta } \\
\text { e demanda. }\end{array}$ \\
\hline $\begin{array}{l}\text { Ausência de } \\
\text { uma das } \\
\text { curvas }\end{array}$ & $\begin{array}{l}\text { - Falhas de codificação (SHAH; } \\
\text { CARPENTER, 1995). } \\
\text { - Dificuldades de processamento da } \\
\text { informação explícita e conceitual } \\
\text { (POSTIGO; POZO, 2000). }\end{array}$ & $\begin{array}{l}\text { - Falta de domínio da noção de equilíbrio } \\
\text { e da distinção entre oferta e quantidade } \\
\text { ofertada, demanda e quantidade } \\
\text { demandada. }\end{array}$ \\
\hline $\begin{array}{l}\text { Ausência de } \\
\text { deslocamento }\end{array}$ & $\begin{array}{l}\text { - Falhas de codificação (SHAH; } \\
\text { CARPENTER, 1995). } \\
\text { - Dificuldades de codificação da } \\
\text { mudança (SHAH; HOEFFNER, 2002). } \\
\text { - Dificuldades de processamento da } \\
\text { informação implícita e conceitual } \\
\text { (POSTIGO; POZO, 2000). }\end{array}$ & $\begin{array}{l}\text { - Falta de domínio dos conceitos de } \\
\text { oferta e demanda e, sobretudo, de seus } \\
\text { respectivos determinantes. } \\
\text { - Incapacidade de distinção da } \\
\text { independência analítica entre oferta e } \\
\text { demanda. }\end{array}$ \\
\hline
\end{tabular}

Quadro 2- Exemplos de pares de rótulos dos eixos apresentadas pelos estudantes Fonte: elaborado pela autora.

O erro mais frequente (36\%), a ausência de aderência com o modelo esperado, expressou-se, por exemplo, com a denominação substancialmente equivocada dos eixos que deveriam conter preço (ordenadas) e quantidades (abscissas). Dentre os pares equivocados registrados pelos estudantes foram identificadas, por exemplo, combinações que constam no Quadro3. 


\begin{tabular}{|c|c|}
\hline Ordenadas ("y") & Abscissas (" $\mathbf{x}$ ") \\
\hline Oferta & Demanda \\
\hline Público alvo & Mercado \\
\hline População & Mercado \\
\hline Mercado de chocolate & \$ cacau \\
\hline Consumidor & Mercado \\
\hline Mercado de transporte público & Entrada do Uber* \\
\hline Mercado de cerveja & Risco de câncer* \\
\hline Y & $\mathbf{x}^{* *}$ \\
\hline \$ & Tempo \\
\hline Preço de mercado & Tempo \\
\hline
\end{tabular}

Quadro 3-Exemplos de pares de rótulos dos eixos apresentadas pelos estudantes Fonte: elaborado pela autora.

O termo "substancialmente equivocada" deve-se aos resultados gráficos associados ao equívoco de rotulagem que inspiraram a construção de gráficos divergentes da proposta, a exemplo da Figura 5:

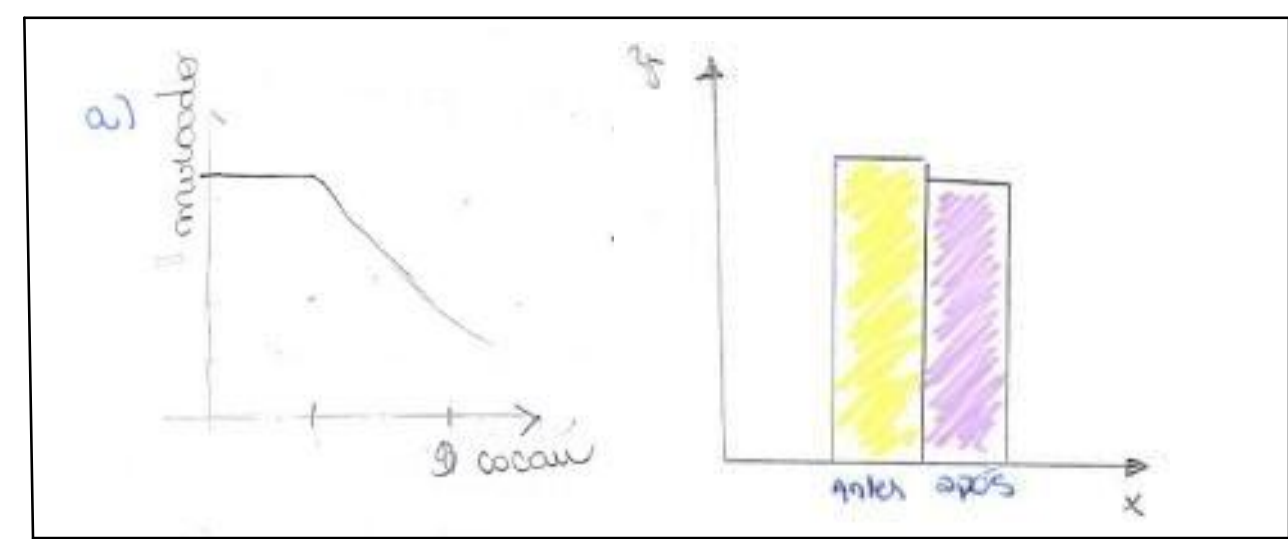

Figura 5 - Exemplo de registro gráfico elaborado por estudante: erros de rotulagem Fonte: elaborada por estudante.

Considerando-se que o modelo de equilíbrio de mercado foi apresentado nas aulas anteriores como gráfico - cartesiano - bidimensional de linha, surpreende a escolha pelo gráfico de barras, dado que tendências do tipo $x-y$ (se "x aumenta, y aumenta", ou se "x aumenta, y diminui") são mais facilmente identificadas em gráficos de linha do que de barras (CARSWELL EMERY; LONON, 1993; ZACKS; TVERSKY, 1999; SHAH et al., 1999). Por outro lado, deve-se destacar a dificuldade metodológica, conceitual - e até mesmo epistemológica - inerente à estática comparativa. Shah e Hoeffner (2002) já haviam mencionado a dificuldade de interpretações qualitativas em gráficos nos quais o tempo não figura como dimensão explícita. Além do gráfico de barras, com indicação explícita de "antes" e "depois", a inclusão do tempo como dimensão explícita no gráfico de outros 5 estudantes (vide exemplo na Figura 6) parece reforçar a hipótese de complexidade de interpretação - e aqui de representação gráfica - da mudança. 


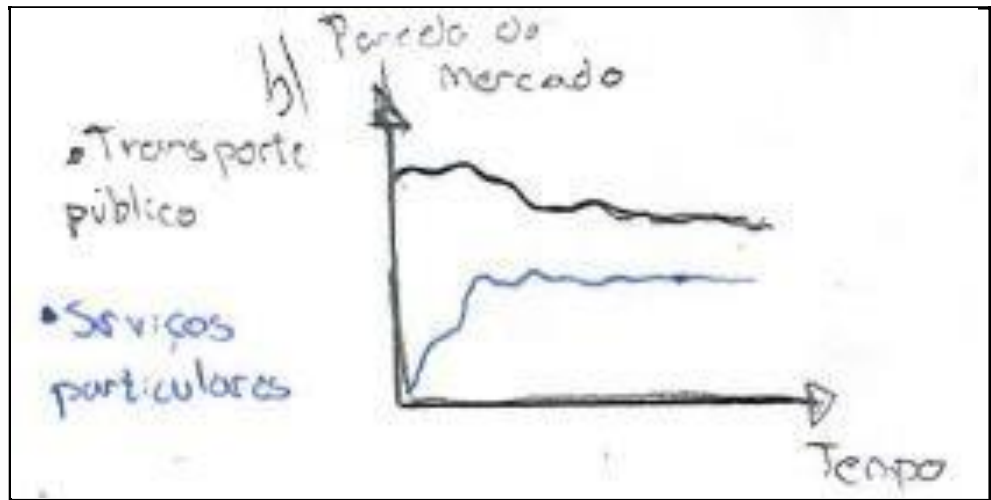

Figura 6 - Exemplo de registro gráfico elaborado por estudante: o tempo como dimensão explícita Fonte: elaborada por estudante.

Os estudantes não parecem estar familiarizados com a estática comparativa enquanto método de representação gráfica da mudança. A estrutura do plano cartesiano, nesse sentido, serviu de suporte para representar tendências lineares (do tipo $x-y$, favorecidas por gráficos de linha) mais relacionadas a fluxo do que à oferta e à demanda no modelo de equilíbrio microeconômico.

Vale ressaltar que a falta de aderência com o modelo de equilíbrio de mercado não implica, necessariamente, incompreensão das relações de causalidade. O exemplo da Figura 7 denota compreensão de causalidade (aumento de custo afeta oferta), mas incapacidade de conversão de causalidade em representação gráfica (esperava-se um deslocamento positivo da curva de oferta).

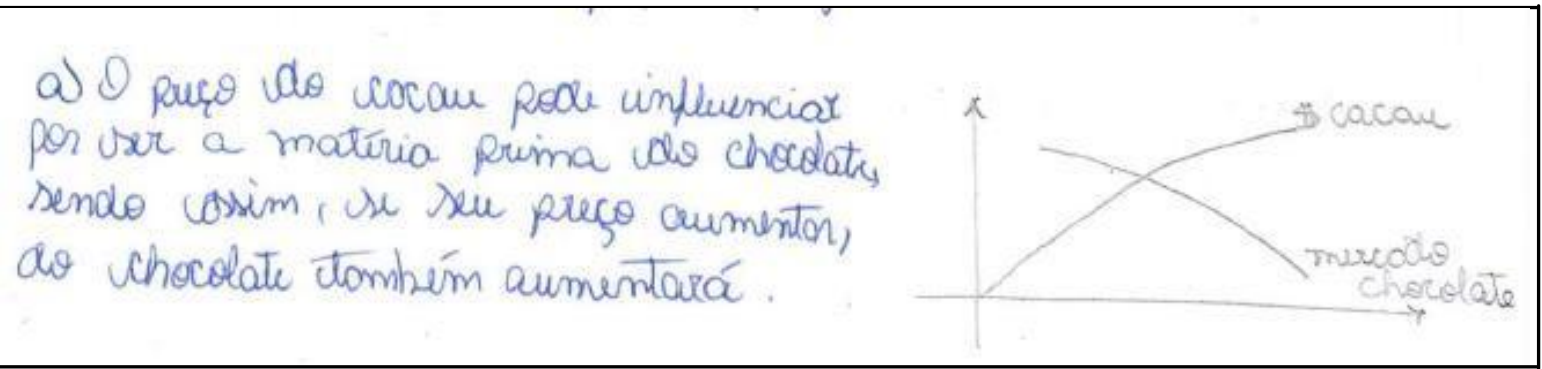

Figura 7 - Exemplo de registro gráfico elaborado por estudante: ausência de aderência Fonte: elaborada por estudante.

Dois estudantes não esboçaram nenhum gráfico. Tal fato indica que eles não tentaram mobilizar conhecimentos prévios (SHAH, 1995), experiência cotidiana ou mesmo intuições (LEINHARDT et al. 1990) no esforço de converter relações funcionais em representações gráficas. Tais relações não se mostraram de todo desconhecidas a esses estudantes, conforme justificativa textual apresentada por um deles para a questão "c" ("a divulgação de tal estudo pode acarretar na queda da demanda por cerveja, forçando os fabricantes a abaixar o preço de venda"). Nos termos de Postigo e Pozo (2000), parece haver dificuldades no que se refere 
Ruth M. Hofmann

ao tratamento da informação implícita, diante da incapacidade de tradução da informação de um código (textual) em outro (gráfico).

Em termos gerais, o conhecimento - ou a sua ausência - dos estudantes sobre gráficos (SHAH; HOEFFNER, 2002) parece ter desempenhado papel importante nos registros apresentados, direcionando as escolhas de tipo e formato adotados.

Os equívocos identificados no deslocamento da curva errada, reflexos de falhas de codificação e de rotulagem associadas a dificuldades de processamento da informação implícita e conceitual (POSTIGO; POZO, 2000), indicam falta de compreensão dos determinantes da oferta e da demanda. Tais erros também guardam semelhança com o erro de deslocamento concomitante das duas curvas (vide Figura 8), embora nesse caso o equívoco conceitual se deva mais à incapacidade de distinção entre quantidade demandada e demanda, quantidade ofertada e oferta. Não há clareza na identificação de relação intra e intervariáveis, por exemplo.

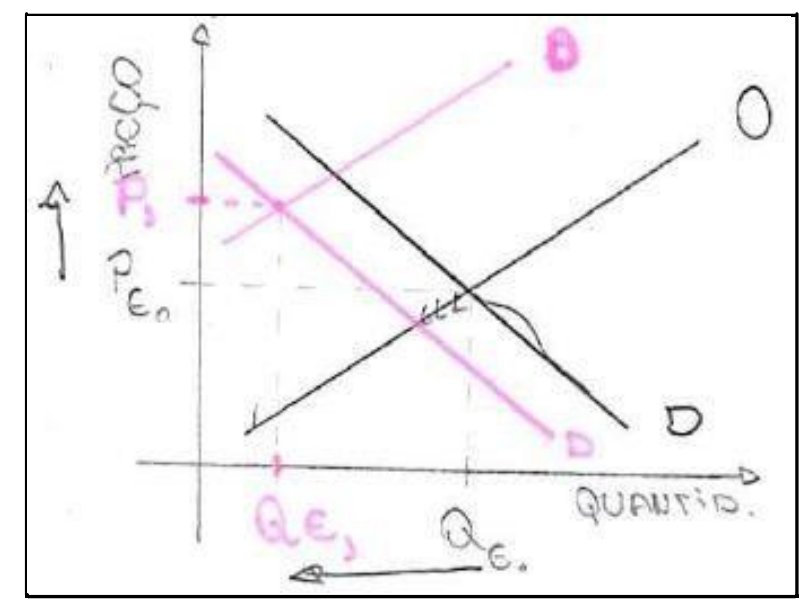

Figura 8 - Exemplo de registro gráfico elaborado por estudante: erro de deslocamento concomitante Fonte: elaborada por estudante.

O equívoco pode estar relacionado à simultaneidade de determinação inerente ao equilíbrio de mercado, o que pode induzir os estudantes a pensar que um único evento deve necessariamente afetar ambas as curvas. Outro fator potencialmente determinante do erro de deslocamento das curvas é a falta de familiaridade com a cláusula coeteris paribus (manter constantes as demais variáveis), recurso analítico de caráter teórico e simplificador que pode divergir substancialmente das intuições e do conhecimento prévio dos estudantes, o que significa que não encontra respaldo nas experiências cotidianas, nas intuições ou no senso comum dos estudantes. A falta de familiaridade com a estática comparativa também deve ser cogitada como fator de determinação dos erros cometidos.

O erro de inversão dos eixos pode ser consequência da frustração de expectativas, relatada por Gattis e Holyoak (1996): os estudantes devem esperar que variáveis dependentes sejam 
Ruth M. Hofmann

plotadas como função de y em um gráfico de linha, e que as variáveis causais ou independentes sejam plotadas como função do eixo y. Como as equações de demanda e de oferta são expressas como $q^{d}{ }_{i=} f\left(p_{i}\right)$ e $q_{i=}^{o} f\left(p_{i}\right)$, erros de inversão entre preço e quantidade como eixos de um gráfico cartesiano são esperados.

Considerando que o trabalho não analisou a compreensão e a construção de gráficos de outra natureza, não é possível afirmar se a origem dos "erros" está em dificuldades habituais dos estudantes na leitura e na construção de gráficos ou se está na fragilidade do ensino de objetos gráficos em sala de aula. A origem pode, ainda, decorrer concomitantemente de ambos. Nesse sentido, cabe o aprofundamento da análise mediante, por exemplo, realização de atividades estruturadas com gráficos de outra natureza, assim como gráficos associados a outros tipos de informação e de contexto. De qualquer forma, uma vez identificadas, as dificuldades apresentadas pelos estudantes fazem necessário o aprimoramento didático da apresentação das representações gráficas de oferta e demanda.

\section{CONSIDERAÇÕES FINAIS}

A Economia, como diversas disciplinas científicas, faz uso dos mais variados tipos de gráficos no processo de divulgação e ensino de conceitos específicos. É o caso do ensino de oferta e demanda, por exemplo. O ensino de Economia, nessa perspectiva, permite o contato reiterado com erros cometidos por estudantes de graduação no processo de construção de representações gráficas clássicas de noções como oferta e demanda. É nesse contexto que o artigo analisou registros de representação gráfica de oferta e demanda construídos por estudantes de Engenharia. Pretendeu-se, assim, identificar desafios cognitivos subjacentes ao ensino de Economia.

Dialogando com a literatura adjacente, em resposta ao primeiro questionamento de Shah e Hoeffner (2002), ou seja, "quais os tipos de erros cometidos na construção de gráficos?", foram identificados equívocos que incluíram desde ausência substancial de aderência com a proposta até equívocos de rotulagem, erros que encontraram respaldo empírico na revisão de literatura e expressaram deficiências de compreensão conceitual. Em resposta ao segundo questionamento, ou seja, "os estudantes escolhem diferentes tipos de gráficos dependendo do objetivo (comunicar fatos específicos, testar uma hipótese etc.) ou eles estão enviesados a usar formatos familiares?", observou-se certa predisposição ao emprego de gráficos nos quais o tempo figura como dimensão explícita no plano cartesiano. Nesse sentido, recorrer a modelos familiares de gráficos de fato parece algo relevante.

Para fins didáticos, as constatações mais relevantes foram a falta de familiaridade com a estática comparativa, recurso metodológico essencial de análise microeconômica. Tal fato, associado à natureza complexa da representação gráfica da mudança (SHAH; HOEFFNER, 
Ruth M. Hofmann

2002), emerge como desafio cognitivo a ser trabalhado em aula. Nesse sentido, convém que o método seja exposto com mais cuidado nas aulas de Economia. A representação do "antes" e "depois" nos gráficos de equilíbrio de mercado, assim, não deve ser tratada superficialmente como pressuposto trivial de análise microeconômica.

Analogamente, a falta de familiaridade com a cláusula "coeteris paribus" e a natureza simultânea da determinação das variáveis envolvidas no modelo de equilíbrio entre oferta e demanda devem ser abordadas sem que se pressuponha tratar-se de elementos simples no processo de ensino de conceitos econômicos. Há desafios cognitivos importantes no ensino de técnicas de análise econômica, incluindo complexidades conceituais e epistemológicas inerentes à disciplina.

Os resultados obtidos incitam reflexões acerca de estratégias didáticas que poderiam ser implementadas para facilitar a leitura e a construção de gráficos por estudantes de economia. Pode-se cogitar, por exemplo:

a) A premissa didática de que os estudantes universitários (mesmo que de Engenharia) estão suficientemente familiarizados com gráficos cartesianos deve ser questionada. Faz-se necessário, por exemplo, realizar uma revisão de funções e gráficos para garantir que os estudantes estejam de fato cientes de sua estrutura e de seu funcionamento.

b) Realizar atividades introdutórias voltadas à identificação do conhecimento prévio dos estudantes acerca de gráficos e representações gráficas, solicitando, por exemplo, que interpretem e discutam dados e informações representados em diferentes formatos.

c) Discutir "gráficos" como objetos em si durante a aula. Especificamente no caso das aulas de Economia, caberia uma discussão sobre os principais tipos, formatos, configurações e convenções gráficas utilizadas para representar relações econômicas. O material de discussão pode incluir gráficos amplamente divulgados em matérias jornalísticas sobre economia e finanças. Discutir os limites e as possibilidades de cada tipo de representação gráfica.

d) Ressaltar a especificidade das convenções gráficas de acordo com diferentes áreas do conhecimento.

e) Ressaltar a relação entre a notação algébrica e a representação gráfica das noções de oferta e demanda.

Deve-se ressaltar que o ensino de conteúdos de determinado campo do conhecimento para estudantes de outras áreas se faz acompanhar de um conjunto de significativos desafios conceituais, metodológicos e didáticos. Cada disciplina científica conta com teorias, conceitos e instrumental metodológico próprio, nem sempre convergindo com abordagens de disciplinas distintas. Nesse sentido, deve-se reconhecer a importância de se assinalar as semelhanças e diferenças conceituais e metodológicas entre as áreas para que os estudantes sejam capazes de construir seu conhecimento tendo ciência das interfaces e das divergências 
Ruth M. Hofmann

entre as disciplinas. Além disso, deve prevalecer a preocupação com a linguagem adotada no ensino. Especificamente no caso da Economia, a julgar pela sequência didática que prevalece nos manuais da disciplina, supõe-se que as representações gráficas sejam mais simples do que a notação algébrica (sendo esta muitas vezes relegada a apêndices). Contudo, os resultados obtidos neste trabalho indicam que essa premissa pode não ser tão robusta. Dependendo da natureza da área de conhecimento dos estudantes, a própria notação algébrica pode ser mais familiar do que representações gráficas abstratas construídas a partir de padrões "pouco convencionais". O exemplo mais emblemático seria a forma eventualmente pouco intuitiva de representação das variáveis econômicas nos eixos das ordenadas e das abscissas no caso de gráficos de oferta e demanda. Trata-se de uma convenção que diverge das práticas de outras áreas de conhecimento e que pode explicar "equívocos" cometidos por estudantes.

\section{REFERÊNCIAS}

CAMARGO FILHO, Paulo Sérgio; LABURU, Carlos Eduardo; BARROS, Marcelo Alves de. Dificuldades semióticas na construção de gráficos cartesianos em cinemática. Caderno Brasileiro de Ensino de Física, Florianópolis, v. 28, n. 3, p. 546-563, 2011.

CARSWELL, C. Melody; EMERY, Cathy; LONON, Andrea. M. Stimulus complexity and information integration in the spontaneous interpretation of line graphs. Applied Cognitive Psychology, n. 7, v. 4, p. 341-357, ago. 1993.

ESTRADA, Mario Arturo Ruiz. Economic graphical modeling from 2-dimensional graphs to multi-dimensional graphs. FEA Working Paper n. 19, 2010. Disponível em: https://ssrn.com/abstract=1367554. Acesso em: 15 maio 2019.

FRANZBLAU, Lauren E.; CHUNG, Kevin C. Graphs, tables, and figures in scientific publications: the good, the bad, and how not to be the latter. The Journal of Hand Surgery, v. 37, n. 3, p. 591-596, 2012.

GALESIC, Mirta; GARCIA-RETAMERO, Rocio. Graph literacy: a cross-cultural comparison. Medical Decision Making. v. 31, p. 444-457, 2011.

GARCÍA GARCÍA, José Joaquín; PERALES PALACIOS, Francisco Javier. ¿Influye la formación acadêmica de los estudiantes en su comprensión de las representaciones gráficas cartesianas? Enseñanza de las Ciencias, España, número extra, VII Congreso, p. 1-5, 2005.

GATTIS, Merideth; HOLYOAK, Keith J. Mapping conceptual to spatial relations in visual reasoning. Journal of Experimental Psychology: Learning, Memory, and Cognition, v. 22, n. 1, p. 231-239, 1996.

LEINHARDT, Gaea; ZASLAVSKY, Orit; STEIN, Mary Kay. Functions, graphs, and graphing: tasks, learning, and teaching. Review of Educational Research, v. 60, n. 1, p. 1-64, 1990.

MOCHÓN, Francisco. Princípios de economia. São Paulo: Pearson Prentice Hall, 2007. 
PARKIN, Michael. Economia. São Paulo: Addison Wesley, 2009.

PASSOS, Carlos Roberto Martins; NOGAMI, Otto. Princípios de economia. 7. ed. São Paulo: Cengage Learning, 2016.

POSTIGO, Yolanda; POZO, Juan Ignacio. Cuando una gráfica vale más que 1.000 datos: la interpretación de gráficas por alumnos adolescentes, Infancia y Aprendizaje. Journal for the Study of Education and Development, v. 23, n. 90, p. 89-110, 2000.

READENCE, John; BEAN, Thomas W.; BALDWIN, Scoth. Content area literacy: an integrated approach, 8 ed. Dubuque, IA: Kendall-Hunt, 2004.

ROBERTS, Kathryn. L.; BRUGAR, Kristy A. The view from here: emergence of graphical literacy. Reading Psychology, v. 38, n. 8, p. 733-777, 2017.

SHAH, Priti. Cognitive process in graph comprehension. Tese de doutorado não publicada. 1995.

SHAH, Priti; CARPENTER, Patricia A. Conceptual limitations in comprehending line graphs. Journal of Experimental Psychology: General, v. 124, n. 1, p. 43-61, 1995.

SHAH, Priti; MAYER, Richard E.; HEGARTY, Mary. Graphs as aid to knowledge construction: signaling techniques for guiding the process of graph comprehension. Journal of Educational Psychology, v. 91, p. 690-702, 1999.

SHAH, Priti; HOEFFNER, James. Review of graph comprehension research: implications for instructions. Educational Psychology Review, n. 1, p. 47-69, mar. 2002.

SOFO, Francesco. Graphic literacy: Part I. A review of the literature. The Vocational Aspect of Education, v. 37, n. 98, p. 107-113, 1985.

VASCONCELLOS, Marco Antônio S. de. Economia: micro e macro. 4. ed. São Paulo: Atlas, 2010.

ZACKS, Jeff; TVERSKY, Barbara. Bars and lines: a study of graphic communication. Memory \& Cognition, v. 27, p. 1073-1079, 1999.

\section{Ruth M. Hofmann}

Possui graduação em Ciências Econômicas, mestrado e doutorado em Educação pela Universidade Federal do Paraná. Atualmente é professora do curso de graduação em Engenharia de Produção da UFPR.

ruthofmann@gmail.com 